\title{
523.
}

\section{ON THE TRANSFORMATION OF UNICURSAL SURFACES.}

[From the Mathematische Annalen, vol. III. (1871), pp. 469-474.]

I CONSIDER the question of the transformation (Abbildung auf einer Ebene) of unicursal surfaces. Taking $(x, y, z, w)$ for the coordinates of a point on the surface, $\left(x^{\prime}, y^{\prime}, z^{\prime}\right)$ for those of the corresponding point on the plane; then if $X^{\prime}, Y^{\prime}, Z^{\prime}, W^{\prime}$ denote each of them a function $\left(x^{\prime}, y^{\prime}, z^{\prime}\right)^{n^{\prime}}$, the equations of transformation are

$$
x: y: z: w=X^{\prime}: Y^{\prime}: Z^{\prime}: W^{\prime}:
$$

and assuming that each of the curves

$$
X^{\prime}=0, \quad Y^{\prime}=0, \quad Z^{\prime}=0, \quad W^{\prime}=0
$$

(or, what is the same thing, the general curve

$$
\left.a X^{\prime}+b Y^{\prime}+c Z^{\prime}+d W^{\prime}=0\right)
$$

passes once through each of $\alpha_{1}$ points, twice through each of $\alpha_{2}$ points, $\ldots, r$ times through each of $\alpha_{r}$ points (for convenience I write $\alpha_{r}$ instead of $\alpha_{r}^{\prime}$ ); and writing also

$$
\begin{aligned}
& n=n^{\prime 2}-\Sigma r^{2} \alpha_{r}, \\
& 0=\frac{1}{2} n^{\prime}\left(n^{\prime}+3\right)-3-\Theta-\Sigma \frac{1}{2} r(r+1) \alpha_{r},
\end{aligned}
$$

(where $\Theta$ is $=0$ or positive except in the case of special relations between the positions of the fixed points $\left.\alpha_{1}, \alpha_{2}, \ldots, \alpha_{r}\right)$, which equations give

$$
-n=3 n^{\prime}-6-2 \Theta-\Sigma r \alpha_{r}
$$

then the order of the surface is $=n$, and the order of the nodal curve is $b=\frac{1}{2}(n-2)(n-3)+\Theta$. I assume that the nodal curve has $h$ apparent double points and $t$ actual triple points, but no stationary points, so that $q$ being the class, we have $q=b^{2}-b-2 h-6 t$; and I endeavour to find these numbers $q, t, h$. 
For this purpose, imagine through the nodal curve a surface of the order $k$, which therefore meets the surface besides in a curve of the order $n k-2 b$; this curve I call the $k$-thic residue of the nodal curve, or simply the "residue." The projection (Abbildung) of the complete intersection $k n$ is a curve of the order $k n^{\prime}$ passing $k r$ times through each of the points $a_{r}$ : this is made up of the projection of the nodal curve once, and of the projection of the residue. But as shown by Dr Clebsch the projection of the nodal curve is of the order $(n-4) n^{\prime}+3$, and it passes $(n-4) r+1$ times through each of the points $\alpha_{r}$; hence the projection of the residue is of the order $(k-n+4) n^{\prime}-3$, and it passes $(k-n+4) r-1$ times through each of the points $\alpha_{r}$. I assume that the projection of the residue is the general curve which satisfies the foregoing conditions, viz. that the residue, and its projection as defined by the foregoing conditions, depend each of them on the same number of constants. The necessity for this is I confess by no means obvious: but take as an illustration Steiner's quartic surface as transformed by the equations $x: y: z: w=x^{\prime 2}: y^{\prime 2}: z^{\prime 2}:\left(x^{\prime}+y^{\prime}+z^{\prime}\right)^{2}:$ the nodal curve consists of three lines meeting in a point, the quadric residue is the remaining intersection of the surface by a quadric cone passing through the three lines; and the projection thereof is a line; the quadric cone, and therefore the conic, each depend upon 2 constants; and the line which is the projection of the conic depends upon the same number (2) of constants: at all events I make the assumption provisionally.

Now in the projection of the residue, we have twice the number of constants

$$
=\left[(k-n+4) n^{\prime}-3\right](k-n+4) n^{\prime}-\Sigma[(k-n+4) r-1](k-n+4) r \alpha_{r},
$$

viz. this is

$$
=(k-n+4)^{2}\left(n^{\prime 2}-\Sigma r^{2} \alpha_{r}\right)+(k-n+4)\left(-3 n^{\prime}+\Sigma r \alpha_{r}\right),
$$

or, what is the same thing, it is

$$
=(k-n+4)^{2} n+(k-n+4)(n-6-2 \Theta),
$$

viz. reducing, and replacing $\Theta$ by its value $=-\frac{1}{2}(n-2)(n-3)+b$, the number in question is

$$
=k^{2} n+k\left(-n^{2}+4 n-2 b\right)+2(n-4) b .
$$

Now $k$ being $=$ or $>n-3$, the curve of intersection of a given surface $n$ by a surface $k$ depends on

$$
\frac{1}{6}(k+1)(k+2)(k+3)-\frac{1}{6}(k-n+1)(k-n+2)(k-n+3)-1
$$

constants; and making the surface $k$ to pass through the curve $b$ we have to subtract herefrom $(k+1) b-\frac{1}{2} g-2 t$; that is, for the residue, twice the number of constants is

$$
=\frac{1}{3}(k+1)(k+2)(k+3)-\frac{1}{3}(k-n+1)(k-n+2)(k-n+3)-2-2(k+1) b+q+4 t,
$$

viz. this is

$$
=k^{2} n+k\left(-n^{2}+4 b-2 b\right)+\frac{1}{3}(n-1)(n-2)(n-3)-2 b+q+4 t .
$$


Hence comparing the two expressions in question we have

that is,

$$
2(n-4) b=\frac{1}{3}(n-1)(n-2)(n-3)-2 b+q+4 t,
$$

$$
0=\frac{1}{3}(n-1)(n-2)(n-3)-2(n-3) b+q+4 t,
$$

or, as I prefer to write it,

$$
0=\frac{1}{6}(n-1)(n-2)(n-3)-(n-3) b+\frac{1}{2} q+2 t ;
$$

which agrees with a more general formula in my "Memoir on the theory of Reciprocal Surfaces," Phil. Trans. vol. CLIX. (1869), [411], see p. 227, [Coll. Math. Papers, vol. vi. p. 356]. I consider any two residues, a $k$-thic residue and a $l$-thic residue; to each intersection of these there corresponds an intersection of their projections: or the number of intersections of the two residues must be equal to that of the two projections. Now the projections being (as above)

order $(k-n+4) n^{\prime}-3$ passing $(k-n+4) r-1$ times through each point $\alpha_{r}$,

$$
\text { ” }(l-n+4) n^{\prime}-3 \quad \text {, }(l-n+4) r-1
$$

the number of the intersections in question is

$$
=\left[(k-n+4) n^{\prime}-3\right]\left[(l-n+4) n^{\prime}-3\right]-\Sigma[(k-n+4) r-1][(l-n+4) r-1] \alpha_{r}+\omega,
$$

where for a reason which will be afterwards explained $I$ have added the term $\omega$ : this is

$$
=(k-n+4)(l-n+4)\left(n^{\prime 2}-\Sigma r^{2} \alpha_{r}\right)+(k+l-2 n+8)\left(-3 n^{\prime}+\Sigma r \alpha_{r}\right)+9-\left(\Sigma \alpha_{r}-\omega\right),
$$

viz. it is

$$
=(k-n+4)(l-n+4) n+(k+l-2 n+8)(n-6-2 \Theta)+9-\left(\Sigma \alpha_{r}-\omega\right),
$$

viz. substituting for $\Theta$ its value, $=-\frac{1}{2}(n-2)(n-3)+b$, and reducing, the number is

$$
=k l n-2(k+l) b-n^{3}+8 n^{2}-16 n+9+4(n-4) b-\left(\Sigma \alpha_{r}-\omega\right) .
$$

But the surfaces $n, k, l$, having in common the curve $b$ which is a nodal curve on $n$, besides intersect in

$$
k l n-b(n+2 k+2 l-4)+2 q+q t
$$

points (Salmon's Geometry of three Dimensions, 2nd Ed. p. 283, except that in the formula as there given the singularity $t$ is not taken account of); that is, the number of intersections of the two residues is

$$
=k l n-2(k+l) b-(n-4) b+2 q+9 t,
$$

which is equal to the number of intersections of the two projections $\left(^{1}\right)$ : or comparing the numbers in question we have

that is,

$$
-n^{3}+8 n^{2}-16 n+9+4(n-4) b-\left(\Sigma \alpha_{r}-\omega\right)=-(n-4) b+2 q+9 t,
$$

$$
2 q+9 t=5(n-4) b-n^{3}+8 n^{2}-16 n+9-\left(\Sigma \alpha_{r}-\omega\right) .
$$

${ }^{1}$ I remark that $n+\lambda$ being positive or not less than $n-3$, two $(n+\lambda)$ thic residues meet in $n(\lambda+4)(\lambda+6)-12 \lambda-39-4(\lambda+4)-\left(\Sigma \alpha_{r}-\omega\right)$ points : in particular, two $(n-3)$-thic residues meet in $3 n-3-4 \theta-\left(\Sigma a_{r}-\omega\right)$ points; and two $(n-2)$-thic residues meet in $8 n-15-8 \theta-\left(\Sigma a_{r}-\omega\right)$ points. 
But we have already found

$$
2 q+8 t=4(n-3) b-\frac{2}{3} n^{3}+4 n^{2}-\frac{22}{3} n+4,
$$

and we have therefore

and

$$
t=(n-8) b-\frac{1}{3} n^{3}+4 n^{2}-\frac{26}{3} n+5-\left(\Sigma \alpha_{r}-\omega\right),
$$

$$
q=-2(n-13) b+n^{3}-14 n^{2}+31 n-18+4\left(\Sigma \alpha_{r}-\omega\right) .
$$

I obtain these results in a different manner by investigating expressions for the deficiency (Geschlecht) of the nodal residue $n k-2 b$ and for that of its projection.

First for the projection, we have

$$
\begin{aligned}
\text { Twice Deficiency } & =\left[(k-n+4) n^{\prime}-4\right]\left[(k-n+4) n^{\prime}-5\right] \\
& -\Sigma[(k-n+4) r-1][(k-n+4) r-2] \alpha_{r}+2 \omega,
\end{aligned}
$$

where I have added the term $2 \omega$, as afterwards explained: this is

$$
=(k-n+4)^{2}\left(n^{\prime 2}-\Sigma r^{2} \alpha_{r}\right)+(k-n+4)\left(-9 n^{\prime}+3 \Sigma r \alpha_{r}\right)+20-2\left(\Sigma \alpha_{r}-\omega\right),
$$

viz. it is

$$
=(k-n+4)^{2} n+(k-n+4)(3 n-18-6 \Theta)+20-2\left(\Sigma \alpha_{r}-\omega\right),
$$

or substituting for $\Theta$ its value $-\frac{1}{2}(n-2)(n-3)+b$ and reducing, it is

$$
=k^{2} n+k\left(n^{2}-4 n-6 b\right)-2 n^{3}+16 n^{2}-32 n+20+6(n-4) b-2\left(\Sigma \alpha_{r}-\omega\right) .
$$

Next as regards the residue, the number $h^{\prime}$ of its apparent double points is obtained in terms of $h$ and $t$ by the formula

$$
8 h+6 t-2 h^{\prime}=(k n-4 b)(k-1)(n-1)-2 b(k-1),
$$

(Salmon, l. c., p. 284, except that the singularity $t$ is not there taken account of); and we thence have

$$
\begin{aligned}
\text { Twice Deficiency } & =(k n-1)(k n-2)-2 h^{\prime} \\
& =k n(k+n-4)+4 b^{2}+b(-4 n-6 k+12)+2-8 h-6 t,
\end{aligned}
$$

or introducing $q$ instead of $h$ by the formula $4 b^{2}-8 h=4 q+4 b+24 t$, this is

viz. it is

$$
=k n(k+n-4)+b(-4 n-6 k+12)+4 q+4 b+2+18 t,
$$

$$
=k^{2} n+k\left(n^{2}-4 n-6 b\right)-4(n-4) b+4 q+18 t .
$$

So that comparing with the deficiency of the projection we have

$$
-2 n^{3}+16 n^{2}-32 n+20+6(n-4) b-2\left(\Sigma \alpha_{r}-\omega\right)=-4(n-4) b+4 q+2+18 t,
$$

that is,

$$
2 q+9 t=5(n-4) b-n^{3}+8 n^{2}-16 n+9-\left(\Sigma \alpha_{r}-\omega\right),
$$

the same result as before. 
The necessity for the term $\omega$ appears by the consideration that if we apply to the plane figure a Cremona-transformation, thus obtaining a new transformation of the surface, the value of $\Sigma \alpha_{r}$ will in general be altered; whereas the expressions for $q, t$ should it is clear remain unaltered; and it arises as follows, viz. for certain transformations of the surface the curve of the order $(k-n+4) n^{\prime}-3$, passing $(k-n+4) r-1$ times through each point $\alpha_{r}$ and assumed to be the projection of the residue, is not an indecomposable curve but contains a certain number $\omega$ of factors (each belonging to a unicursal curve definable by means of the number of its passages through the several points $\alpha_{r}$ ), which factors are to be rejected in order to obtain the equation of the proper residue. Thus reverting to the transformation

$$
x: y: z: w=x^{\prime 2}: y^{\prime 2}: z^{\prime 2}:\left(x^{\prime}+y^{\prime}+z^{\prime}\right)^{2}
$$

of Steiner's surface, the projection of the quadric residue was (as already remarked) a line; applying to the plane figure the ordinary quadric (or inverse) transformation we introduce three fixed points, $\left(\alpha_{2}=3\right)$, say these are $A, B, C$; viz. in the new transformation of the surface the projection of any plane section is a quartic curve having a node at each of the fixed points: the projection of the residue ought clearly to be a conic through the three points; but according to the general formula it is a quintic having at each of these points a triple point: the quintic is in fact made up of the lines $B C, C A, A B$ and of the conic which is the proper residue; viz. in the case in question there are 3 factors thrown out, or we have $\omega=3$. To apply this to the second investigation of $2 q+9 t$, by comparison of the two deficiencies, observe that in general if a curve is made up of $\omega+1$ indecomposable curves, the deficiency of the compound curve is equal to the sum of the deficiencies of the component curves $-\omega$; hence if $\omega$ of the curves are unicursal, the deficiency of the compound curve is equal to that of the remaining curve $-\omega$; or, what is the same thing, the deficiency of the remaining curve is $=$ that of the compound curve $+\omega$; and the addicion of the term $+\omega$ to the expression for the deficiency is thus accounted for. It is easy to see that a like explanation applies to the first investigation of $2 q+9 t$.

I further remark, reverting to the equations

$$
x: y: z: w=X^{\prime}: Y^{\prime}: Z^{\prime}: W^{\prime}
$$

of the transformation, that the product of the $\omega$ factors is given as the common factor (if any) of the Jacobians

$$
J\left(Y^{\prime}, Z^{\prime}, W^{\prime}\right), \quad J\left(Z^{\prime}, W^{\prime}, X^{\prime}\right), \quad J\left(W^{\prime}, X^{\prime}, Y^{\prime}\right) \text { and } J\left(X^{\prime}, Y^{\prime}, Z^{\prime}\right) .
$$

Such common factor exists whenever we can by a Cremona-transformation of the plane figure reduce the number of the points $\alpha_{r}$ upon which the transformation of the surface depends; viz. for any given transformation of the surface, $\omega$ is equal to the excess of $\Sigma \alpha_{r}$ above the minimum value of $\Sigma \alpha_{r}$, or, what is the same thing, $\Sigma \alpha_{r}-\omega$ is equal to the minimum value of $\Sigma \alpha_{r}$, and is thus independent of the particular transformation. And of course if $\Sigma \alpha_{r}$ has this minimum value, viz. if the transformation is such that the number of the points $\alpha_{r}$ cannot be reduced by any Cremona-trans- 
formation of the plane figure, then we have $\omega=0$. I presume that for the most simple transformation, that is, when $n^{\prime}$ has its least value, $\Sigma \alpha_{r}$ has also its least value, and consequently that $\omega$ is $=0$.

Recapitulating, the results obtained are

$$
\begin{aligned}
& q=-2(n-13) b+n^{3}-14 n^{2}+31 n-18+4\left(\sum \alpha_{r}-\omega\right), \\
& t=(n-8) b-\frac{1}{3} n^{3}+4 n^{2}-\frac{26}{3} n+5-\left(\sum \alpha_{r}-\omega\right),
\end{aligned}
$$

where it will be recollected that

$$
b=\frac{1}{2}(n-2)(n-3)+\Theta ;
$$

the formulæ are verified in the several cases:

\begin{tabular}{|c|c|c|c|c|c|c|c|c|l|}
\hline$n^{\prime}$ & $a_{1}$ & $a_{2}^{\prime}$ & $n$ & $\Theta$ & $\omega$ & $b$ & $q$ & $t$ & \\
\hline 2 & 2 & 0 & 2 & 0 & 1 & 0 & 0 & 0 & Quadric surface \\
\hline 2 & 1 & 0 & 3 & 1 & 0 & 1 & 0 & 0 & Cubic scroll \\
2 & 0 & 0 & 4 & 2 & 0 & 3 & 0 & 1 & Steiner's quartic surface \\
3 & 6 & 0 & 3 & 0 & 0 & 0 & 0 & 0 & Cubic surface \\
3 & 5 & 0 & 4 & 1 & 0 & 2 & 2 & 0 & Quartic with nodal conic \\
2 & 8 & 1 & 4 & 0 & 0 & 1 & 0 & 0 & Do. with nodal line \\
2 & 7 & 1 & 5 & 1 & 0 & 4 & 8 & 0 & Quintic with nodal quadriquadric \\
2 & 11 & 0 & 5 & 0 & 0 & 3 & 4 & 0 & Do. with nodal skew cubic \\
2 & 12 & 2 & 5 & -1 & 0 & 2 & 0 & 0 & Do. with two non-intersecting nodal lines \\
\hline
\end{tabular}

which are the transformations chiefly as yet examined: but the first-mentioned case (quadric surface, generalised stereographic projection), although as stated the formulæ are verified with the value $\omega=1$, does not really come under the foregoing theory. It is interesting to see that they are verified in the last-mentioned case, belonging to a negative value of $\Theta$, that is, to a special system of fixed points.

Cambridge, Dec. 5, 1870.

C. VIII. 